\section{An algorithm to generate discrete probability distributions: Binomial, hypergeometric, negative binomial, inverse hypergeometric, and Poisson}

\author{
KENNETH J. BERRY, PAUL W. MIELKE, JR., and \\ STEVEN G. HELMERICKS \\ Colorado State University, Fort Collins, Colorado
}

A recursion procedure for generating point probability values, cumulative probability values, and interval probability values of discrete distributions is described. Algorithms and associated FORTRAN functions for five representative discrete probability distributions are presented: binomial, hypergeometric, negative binomial, inverse hypergeometric, and Poisson. The joint use of recursion and a small arbitrary initial value ensures computational efficiency.

A recursive technique for generating point probability values, cumulative probability values, and interval probability values of discrete distributions is presented. Functions for five representative discrete distributions are described: binomial, hypergeometric, negative binomial, inverse hypergeometric, and Poisson. Detailed descriptions of these discrete probability distributions are presented in reference sources such as Parzen (1960), Feller (1968), Johnson and Kotz (1969), and Tsokos (1972).

Each of the five discrete distributions is composed of point probabilities involving factorial expressions, which are often difficult to compute. A recursion technique eliminates the calculation of all factorial expressions by generating relative frequencies that are proportional to the required probability values. The result is a set of highly efficient distribution functions, producing point, cumulative, or interval probability values. These functions are extremely fast, highly accurate, and require very little machine memory. The algorithm common to all five distribution functions incorporates both mathematical recursion and an arbitrary initial value, generating a choice of point, cumulative, or interval probability values. Although recursion algorithms are common, a survey of the recent literature regarding statistical and probability algorithms failed to yield this algorithm or its variants. Specifically, popular collections of statistical algorithms and reference sources for statistical algorithms were scrutinized (e.g., Cormen, 1990; Griffiths \& Hill, 1985; Knuth, 1969; Kohn, 1987; Mardia \& Zem-

Address correspondence to K. J. Berry, Department of Sociology, Colorado State University, Fort Collins, CO 80523 (e-mail: berry@) lamar.colostate.edu).

$$
\text { -Accepted by previous editor, N. John Castellan, Jr. }
$$

roch, 1978; Pooch \& Wall, 1993; Press, Flannery, Teukolsky, \& Vetterling, 1986; Sedgewick, 1988; Tsokos, 1972).

Since the binomial, hypergeometric, and inverse hypergeometric are closed-sum probability distributions, and the negative binomial and Poisson are open-sum probability distributions, only one representative distribution from each class is described below: the binomial and the negative binomial.

\section{Binomial Distribution}

The binomial probability function,

$$
p(x \mid n, \pi)=\left(\begin{array}{l}
n \\
x
\end{array}\right) \pi^{x}(1-\pi)^{n-x},
$$

gives the point probability, $p$, of obtaining $x$ marked items in a random sample of $n$ marked and unmarked items, drawn with replacement, with the population proportion of marked items designated by $\pi$. If $r(x \mid n, \pi)$ is a recursively defined positive function, and $r(x=$ $0 \mid n, \pi)$ is an arbitrary positive constant, then

$$
r(x+1 \mid n, \pi)=r(x \mid n, \pi) \cdot \frac{(n-x) \pi}{(x+1)(1-\pi)},
$$

and the cumulative probability, $P$, of $x$ marked items is given by

$$
P(x \mid n, \pi)=\sum_{j=0}^{x} r(j \mid n, \pi) / \sum_{j=0}^{n} r(j \mid n, \pi) .
$$

It is interesting to note that the first person to use this technique to calculate binomial probability values was Quetelet (1849), over 140 years ago. Because of the recursive structure of these algorithms, the probability of the binomial random variable's being in the inclusive interval from $x_{1}$ to $x_{2}$ is given by

$$
P\left(x_{2} \mid n, \pi\right)-P\left(x_{1}-1 \mid n, \pi\right) \text {. }
$$

\section{Negative Binomial Distribution}

The negative binomial probability function,

$$
p(x \mid m, \pi)=\left(\begin{array}{c}
x+m-1 \\
m-1
\end{array}\right) \pi^{m}(1-\pi)^{x},
$$

gives the point probability, $p$, of $x$, where $m>0$ is the negative binomial intensity parameter and $0<\pi<1$. If $m$ is a positive integer, then $x$ and $m$ are the number of unmarked and marked items in the sample, respectively, and $\pi$ is the proportion of marked items in the population. If $r(x \mid m, \pi)$ is a recursively defined positive function, and $r(x=0 \mid m, \pi)$ is an arbitrary positive constant, then 


$$
r(x+1 \mid m, \pi)=r(x \mid m, \pi) \cdot \frac{(x+m)(1-\pi)}{x+1},
$$

and the cumulative probability, $P$, of $x$ unmarked items is given by

$$
P(x \mid m, \pi)=\sum_{j=0}^{x} r(j \mid m, \pi) / \sum_{j=0}^{w} r(j \mid m, \pi) .
$$

Here, $w$ is the maximum of $\mu+G \sigma, x+G \sigma$, or $x+10$, where $G=40$ when $\mu<1, G=30$ when $1 \leq \mu<16$, $G=20$ when $\mu \geq 16$, and $\mu$ and $\sigma$ are the exact mean and standard deviation of the negative binomial random variable. The probability of the negative binomial random variable's being in the inclusive interval from $x_{1}$ to $x_{2}$ is given by $P\left(x_{2} \mid m, \pi\right)-P\left(x_{1}-1 \mid m, \pi\right)$; here, $w$ is the maximum of $\mu+G \sigma, x_{2}+G \sigma$, or $x_{2}+10$.

\section{System Requirements and Language}

The five functions (BINOML, HYPERG, NEGBIN, INVHYP, and POISSN) are written in ANSI-standard FORTRAN-77 in double precision. Comment lines provide documentation and specification of dummy arguments and output.

\section{Testing and Verification}

The five functions were tested and verified by using a variety of analytical and calculation methods. In addition, selected results of the functions were verified by comparing the results with values in one or more published sets of tables, including Tables of the Hypergeometric Probability Distribution (Lieberman \& Owen, 1961); Tables of the Cumulative Binomial Probability Distribution for Small Values of $p$ (Weintraub, 1963); Tables of the Negative Binomial Probability Distribution (Williamson \& Bretherton, 1963); Tables of the Individual and Cumulative Terms of Poisson Distribution (General Electric Co., 1962); 50-100 Binomial Tables (Romig, 1947); and Binomial, Normal, and Poisson Probabilities (Smith, 1953). All results were calculated on a 486-DX personal computer and on an IBM RISC System/6000 Model 980 , in double precision. The verification for the binomial distribution included selected values of the population proportion $p$ and the sample size $n$ over the ranges of $p=.00001$, with $n=1$ up to $p=.50$ with $n=100$. The verification for the hypergeometric distribution included selected values of the population size and the sample size over the ranges of a population of size 2 with a sample of size 1 , up to a population of size 1,000 with a sample of size 500 . The verification for the negative binomial included selected values of the population proportion $\pi$ and the negative binomial intensity parameter $m$ over the ranges of $\pi=$ 0.05 and $m=0.1$ up to $\pi=0.95$ with $m=200$. The verification for the inverse hypergeometric distribution included selected values of the population size and the sample size over the ranges of a population of size 2 with a sample of size 1, up to a population of size 5,000 with a sample of size 4,000 . The verification for the Poisson distribution included selected values of the Poisson intensity parameter and the number of counts over the ranges of an intensity parameter of 0.0000001 with counts of 0 and 1 , up to an intensity parameter of 205 with counts from 123 to 289 .

\section{Availability}

Listings of the five functions and an appropriate driver program are available from Kenneth J. Berry, Department of Sociology, Colorado State University, Fort Collins, CO 80523 (e-mail: berry@lamar.colostate. edu).

\section{REFERENCES}

CORMEN, T. H. (1990). Introduction to algorithms. New York: McGrawHill.

FELLER, W. (1968). An introduction to probability theory and its applications (3rd ed.). New York: Wiley.

General Electric Company (1962). Tables of the individual and cumulative terms of Poisson distribution. New York: Van Nostrand.

GRIFFITHS, P., \& Hill, I. D. (1985). Applied statistics algorithms. Chichester: Horwood.

Johnson, N. L., \& KoTZ, S. (1969). Discrete distributions. Boston: Houghton-Mifflin.

KNUTH, D. E. (1969). The art of computer programming: Vol. 2. Seminumerical algorithms. Reading, MA: Addison-Wesley.

KoHN, M. C. (1987). Practical numerical methods: Algorithms and programs. New York: MacMillan.

Lieberman, G. J., \& OWEN, D. B. (1961). Tables of the hypergeometric probability distribution. Stanford: Stanford University Press.

MARDIA, K. V., \& ZEMROCH, P. J. (1978). Tables of the F-and related distributions with algorithms. New York: Academic Press.

PARZEN, E. (1960). Modern probability theory and its applications. New York: Wiley.

Pooch, U. W., \& WALL, J. A. (1993). Discrete event simulation. Boca Raton, FL: CRC Press.

Press, W. H., Flannery, B. P., Teukolsky, S. A., \& Vetterling, W. T. (1986). Numerical recipes: The art of scientific computing. New York: Cambridge University Press.

QUeTELET, M. A. (1849). Letters addressed to H.R.H., the Grand Duke of Saxe Coberg, and Gotha on the theory of probabilities as applied to the moral and political sciences ( $\mathrm{O}$. G. Downes, Trans.). London: Charles \& Edwin Layton.

Roмig, H. G. (1947). 50-100 binomial tables. New York: Wiley.

SEDGEWICK, R. (1988). Algorithms. Reading, MA: Addison-Wesley.

SMITH, E. S. (1953). Binomial, normal, and Poisson probabilities. Bel Air, MD: E. S. Smith.

Tsokos, C. P. (1972). Probability distributions: An introduction to probability theory with applications. Belmont, CA: Duxbury Press.

WeInTRAUB, S. (1963). Tables of the cumulative binomial probability distribution for small values of p. New York: Free Press of Glencoe.

Williamson, E., \& BRETHERTON, M. H. (1963). Tables of the negative binomial probability distribution. New York: Wiley.

(Manuscript received November 23, 1992; revision accepted for publication November 15,1993 .) 